\title{
Proteomics Analysis of Differential Expression of Lung Proteins In Response To Highly Pathogenic Avian Influenza Virus Infection In Chicken
}

\section{Periyasamy Vijayakumar}

ICAR - National Institute of High Security Animal Diseases: National Institute of High Security Animal Diseases

\section{Ashwin Ashok Raut}

ICAR - National Institute of High Security Animal Diseases: National Institute of High Security Animal Diseases

\section{Santhalembi Chingtham}

ICAR - National Institute of High Security Animal Diseases: National Institute of High Security Animal Diseases

\section{Harshad V Murugkar}

ICAR - National Institute of High Security Animal Diseases: National Institute of High Security Animal Diseases

\section{Diwakar D. Kulkarni}

ICAR - National Institute of High Security Animal Diseases: National Institute of High Security Animal Diseases

\section{Vijendra Pal Singh}

ICAR - National Institute of High Security Animal Diseases: National Institute of High Security Animal Diseases

\section{Anamika Mishra ( $\nabla$ reach2anamika@yahoo.com )}

ICAR - National Institute of High Security Animal Diseases https://orcid.org/0000-0001-5954-3905

\section{Research Article}

Keywords: Avian influenza virus, proteome, molecular pathways, proteomic determinants, chicken, lung tissues

Posted Date: June 30th, 2021

DOl: https://doi.org/10.21203/rs.3.rs-573195/v1

License: (a) This work is licensed under a Creative Commons Attribution 4.0 International License. Read Full License 
Version of Record: A version of this preprint was published at Archives of Virology on November 16th, 2021. See the published version at https://doi.org/10.1007/s00705-021-05287-5. 


\section{Abstract}

Elucidation of molecular pathogenesis underlying virus-host interaction is important for the development of new diagnostic and therapeutic strategies against highly pathogenic avian influenza (HPAI) infection in chicken. However, chicken HPAl viral pathogenesis is not completely understood. To elucidate the intracellular signaling pathways and critical host proteins associated with influenza pathogenesis, we characterized the lung proteome of chicken infected with HPAI H5N1 virus (A/duck/India/02CA10/2011/Agartala). The chicken mass spectra data sets comprised1, 47, $451 \mathrm{MS}$ scans and 19, 917 MS/MS scans. At local FDR 5\% level, we identified total 3313 chicken proteins with presence of at least one unique peptide. At $12 \mathrm{hrs}, 247$ proteins are downregulated while 1754 proteins are downregulated at $48 \mathrm{hrs}$ indicating that the host has succumbed to infection. There is expression of proteins of the predominant signaling pathways, such as TLR, RLR, NLR and JAK-STAT signaling. Activation of these pathways is associated with cytokine storm effect and thus may be the cause of severity of HPAI H5N1 infection in chicken. Further we identified proteins like MyD88, IKBKB, IRAK4, RELA, and MAVS involved in the critical signaling pathways and some other novel proteins (HNF4A, ELAVL1, FN1, COPS5, CUL1, BRCA1 and FYN) as main hub proteins that might play important roles in influenza pathogenesis in chicken. Taken together, we characterized the signaling pathways and the proteomic determinants responsible for disease pathogenesis in chicken infected with HPAI H5N1 virus.

\section{Introduction}

The control measures to any emerging and re-emerging infection, requires an adequate understanding of virus-host interaction [1]. The complete understanding of virus-host interaction will provide important clues for the development of new diagnostic and therapeutic strategies against emerging diseases. The high-throughput functional genomics approach provides a deeper understanding of emerging disease by encompassing both the pathogen and the host response. Virus-host interactions are multidimensional in nature, and this interaction alters various host components such as transcriptome, proteome, miRNA, metabolome, and lipidome [2]. In past years, most of the high-throughput studies on H5N1-host interactions have focused at the transcriptome level.

However, transcriptomic studies do not reveal any posttranscriptional regulation, posttranslational modifications, and protein-protein interactions [3]. The virus-host interaction involves the alternation of hundreds to thousands of host proteins. Hence, applying high throughput proteomics approach for the analysis of virus-induced innate immune responses in conjunction with the transcriptomic analyses promises better understanding of molecular mechanism of influenza pathogenesis [4]. Proteomics refers to the large-scale study of protein expression, protein-protein interactions or posttranslational modifications based on high-resolution mass spectrometry $[5,6]$.

Proteomics studies of influenza virus infection in macaques [7], mice [8], continuous cell lines [9-11], chicken [12,13], dogs [14] and primary human cells [15-18] have provided information on alteration of host proteome at cellular and organism levels. Recent studies of chicken proteome response against 
H5N1 infection revealed several alterations of cytoskeleton, metabolic process, cellular component, and transcription regulation proteins $[12,13]$. However, detailed knowledge of signalling pathways and proteomic determinants responsible for the HPAI H5N1 viral pathogenesis in avian species is not known. In this study, we characterized the signaling pathways and the proteomic determinants responsible for disease pathogenesis in chicken infected with HPAI H5N1 virus at the proteome level.

\section{Materials And Methods}

\section{Experimental infection of chicken}

Six weeks old healthy domestic chicken, sero-negative for Avian Influenza virus (AIV) were used for this study. The chicken obtained from Specific Pathogen Free hatchery unit of ICAR-National Institute of High Security Animal Diseases, Bhopal, India. The animal experiments were approved by the Institutional Animal Ethics Committee of ICAR-NIHSAD (Approval no. 68/IAEC/HSADL/12 dated 11.05.2012) and all the experiments were conducted in biosafety level 3 containment facility of ICAR-National Institute of High Security Animal Diseases, Bhopal, India. The chicken was separated into four groups $(n=5$ birds/group). Among the four groups, three groups were intranasally inoculated with $10^{6} \mathrm{EID}_{50}$ of H5N1 virus (A/duck/India/02CA10/2011/Agartala) and one group (control) was inoculated with PBS. The birds were observed daily for clinical signs. All birds were euthanized by cervical dislocation. Lung tissues were collected from five birds from each infected group at $12 \mathrm{hr}, 24 \mathrm{hr}$, and $48 \mathrm{hr}$ post-infection. Lung tissues were collected from the control group at $12 \mathrm{hr}$ post-inoculation. The tissues were snap chilled in Liquid Nitrogen and stored at $-80^{\circ} \mathrm{C}$ until protein extraction. Avian influenza virus infection of lung tissues was confirmed by virus isolation upon inoculation in embryonated chicken eggs and RT-PCR.

\section{Protein extraction}

$150 \mathrm{mg}$ lung tissue from each sample was washed in $50 \mathrm{mM} \mathrm{NH}_{4} \mathrm{HCO}_{3}$ washing buffer. The lung tissue was cut into small pieces and $650 \mu$ of SDS protein extraction lysis buffer [0.1\% SDS (Invitrogen); 50mM $\mathrm{NH}_{4} \mathrm{HCO}_{3}$ (Sigma); $1 \mathrm{X}$ cOmplete ${ }^{\mathrm{TM}}$ Protease Inhibitor Cocktail (Roche-11836145001)] was added. Tissue samples were homogenized in LZ-Lyser homogenizer at $30 \mathrm{HZ}$ for 2 min. After complete homogenization, the tissue lysate was incubated on ice for 90 mins for complete protein lysis. The lysate was centrifuged at $20,000 \mathrm{~g}$ for $60 \mathrm{mins}$ at $4^{\circ} \mathrm{C}$ and the supernatant was collected. The supernatants were immediately snap heat treated at $56^{\circ} \mathrm{C}$ for $30 \mathrm{~min}$ in a dry bath for inactivation of HPAIV H5N1 in the protein extracts. All the heat-treated samples were stored at $-80^{\circ} \mathrm{C}$ for mass spectrometry analysis.

\section{Sample preparation for LC-MS analysis}

Lung protein quality was checked by $8 \%$ SDS-PAGE. A pool for each time point was prepared by pooling $50 \mu \mathrm{g}$ protein each from 3 best samples at that time point. Protein samples were reduced for $1 \mathrm{hr}$ at $95^{\circ} \mathrm{C}$ in $100 \mathrm{mM}$ dithiothreitol solution followed by alkylation for $45 \mathrm{~min}$ by $55 \mathrm{mM}$ iodacetamide in the dark at RT. Trypsin was added to all protein samples at a 1:20 (wt/wt) trypsin-to-protein ratio for overnight at $37^{\circ} \mathrm{C}$. After trypsin digestion, the sample quality was again checked by SDS PAGE. Digested peptide 
samples were concentrated to $50 \mu$ total volume using a centrifugal vacuum. The non-infected and infected peptide samples were labeled with iTRAQ 4-Plex (P/N: 4352135) reagents. The labels used for sample pools of different groups were as follows: iTRAQ label 114- control; iTRAQ label 115-12hr; iTRAQ label 116-24hr; iTRAQ label 117-48hr. These iTRAQ labeled samples were pooled and then purified using a strong cation exchange (SCX) chromatography. The fractions from SCX chromatography were collected and pooled according to RT values. The pooled fractions were vacuum dried and dissolved in $10 \mu \mathrm{L}$ of $0.1 \%$ formic acid. The sample was injected $(1 \mu \mathrm{L})$ onto $\mathrm{C} 18$ Nano-LC column for separation of peptides followed by mass spectrometry analysis on the WATERS Q-TOF instrument.

\section{Bioinformatics analysis}

The WATRES specific raw data set files were converted to proteomics standard format mzXML format by proteowizard tool MSconvert with default parameters option [19]. The database search and other downstream bioinformatic analysis were done in Trans-Proteomic Pipeline (TPP) [20]. TPP includes modules for validation of database search results, quantitation of isotopically labeled samples, and validation of protein identifications. MS/MS ion spectra were searched against chicken UniProt reference database using comet search engine [21]. All 17719 proteins that comprise the chicken UniProt reference database and 11 protein sequences of HPAIV H5N1 virus (A/duck/India/02CA10/2011/Agartala from NCBI protein database were downloaded. The following parameters were used for database search, precursor/peptide mass tolerance-1.8Da, fragment tolerance $-1.6 \mathrm{Da}$, fixed modification carboxamidomethylation for cys (C) and iTRAQ $N$ terminal, Variable modification- oxidation (M) and phosphorylation of (STY), and number of missed cleavages 2. The Peptide-Spectrum assignments of comet search engine were validated with PeptideProphet and iProphet tool of TPP [22]. Protein identifications were validated with ProteinProphet tool of TPP based on PeptideProphet or iProphet results [23]. Proteins identified from ProteinProphet result were filtered based on protein probability above 0.95 (local FDR $5 \%$ level) and contained at least 1 unique peptide. This filtered protein list was used for further downstream functional analysis. Up and downregulation of particular protein was calculated as infected sample intensity divided by control sample intensity (i.e. 115-12hr/114-control). Likewise for all proteins and specific post-infection time interval, fold change values were calculated. Functional classification of the proteins was performed for gene ontology (GO) in Database for Annotation, Visualization and Integrated Discovery (DAVID) [24] and pathway analysis in Kyoto Encyclopedia of Genes and Genomes (KEGG) (www.genome.jp/kegg/). Heatmap generated using 'Clustvis' web tool [25]. We used online web server NetworkAnalyst for construction of protein-protein interaction (PPI) networks [26]. The main driving or hub proteins were identified on the basis of two topological measures, degree centrality and betweenness centrality.

\section{Meta-analysis of transcriptome datasets}

For meta-analysis of chicken transcriptome datasets, we selected the microarray datasets of 2 independent studies. Hu et al. (2015) studied immune response of primary chicken lung cells infected with two HPAI H5N1 viruses using microarray technology [27]. Ranawareet al. (2016) studied global immune response of chicken infected with HPAI H5N1 (A/duck/India/02CA10/2011) virus infection [28]. 
The microarray datasets were analysed in GEO2R online tool of the NCBI. The original submitter-supplied processed microarray data tables were identified using the GEO query. Then, we identified control and test group samples and then samples belonging to each group were assigned. Further, the logFC, p-value and adj. p-value were calculated by limma R package. Adjusted p-values below 0.05 were used as threshold to find differentially expressed genes.

Bayesian networks were reconstructed for TLR, RLR, IL1R, NLR and JAK-STAT signaling pathways using 'bnlearn' package [29]. The networks were constructed based on intensity values of microarray dataset of our pervious published work [28]. Bayesian network structure was learned from transcriptomic dataset with prior knowledge using Hill Climbing $(\mathrm{HC})$ algorithm. $\mathrm{HC}$ is a score-based structure learning algorithm. After learning the network structure, the conditional probability tables (CPTs) at each node were found by running bn.fit function. Bn.fit runs the EM algorithm to learn CPT for different nodes in the learned graph.

\section{Results And Discussion}

Virus-host interactions are multidimensional, they include the alterations in transcriptome, proteome, metabolome and lipidome of the host. In recent years, most of the high-throughput studies have focused majorly on the host transcriptomic responses and other host components have received less attention. This study presents a comprehensive characterization of the lung proteome, critical signaling pathways and proteomic determinants responsible for disease pathogenesis at the proteome level in chicken lung tissues infected with HPAI H5N1 virus over different time points post-infection.

\section{Clinical signs}

Chicken in the control group did not show any clinical signs during the experimental period. In the test group, the birds were normal up to $12 \mathrm{hr}$ post-infection. Mild clinical symptoms like depression, decreased feed as well as water consumption and ruffled feathers were observed at $24 \mathrm{hr}$ post-infection. The clinical symptoms like dullness, lacrimation, cyanotic combs and wattles, edema and red discoloration of the shanks and feet were seen in the birds at $48 \mathrm{hr}$ post-infection.

\section{Raw mass spectra dataset analysis}

Total 15 fraction s of WATERS QTOP raw data sets were generated, with data size 48 GB, including 1 , 47,451 MS scans and 19,917 MS/MS scans. Total 19,917 MS/MS spectra were searched against chicken protein database by comet search engine. The iProphet algorithm identified 17,273 unique peptides and 8,516 unique proteins at minimum probability threshold of above 0.05 and minimum above 7 amino acids. In order to increase the accuracy of validation of peptides and proteins, we applied local FDR 5\% level (probability cut off above 0.95 ) in the ProteinProphet output. At higher probability threshold cut off, we identified total 3313 proteins with the presence of at least one unique peptide. ProteinProphet predicted sensitivity and error rate information is shown in Fig.S1. This is the highest protein identification reported till date in chicken. Our proteomic approach identified H5N1 viral peptides such as NA, NP and PB1 in the chicken lung proteome. NA is a sialidase responsible for releasing sialic acid from 
glycoprotein and glycolipid sialoconjugates of bound influenza virus to assist virus release [30]. NP is an important viral protein responsible for the packaging of the viral RNA and also shown to be involved in many aspects of influenza viral replication [31,32]. PB1 viral protein has been shown to be associated with the high pathogenicity of H5N1 viruses infection in ducks [33]. Influenza viral infection status of lung tissues was confirmed by virus isolation in embryonated egg inoculation and RT-PCR method as well as by identification of viral peptides in lung proteome dataset.

\section{Differential protein expression analysis}

At $12 \mathrm{hr}$ post-infection, 820 proteins were upregulated and 2493 proteins were downregulated in chicken lung tissues. A total of 827 proteins were upregulated and 2441 proteins were downregulated at $24 \mathrm{hr}$ post-infection. At $48 \mathrm{hr}$ post-infection, 693 proteins were upregulated and 2620 proteins were downregulated in chicken lung proteome (Table 1). A total of 470 and 2235 proteins were found to be commonly upregulated and downregulated in all time intervals post-infection in chicken lungs infected with H5N1 virus, respectively (Fig. 1). The protein profile showed that 70, 70 and 101 proteins were exclusively up regulated in chicken lung tissue at $12 \mathrm{hr}, 24 \mathrm{hr}$ and $48 \mathrm{hr}$ post-infection, respectively (Fig. 1). Downregulation of 87,35 and 245 proteins were observed exclusively at $12 \mathrm{hr}, 24 \mathrm{hr}$ and $48 \mathrm{hr}$ postinfection, respectively (Fig. 1). The fold change value of the upregulated proteins ranged from 42 to 1. Interestingly, at $48 \mathrm{hr}$ time point (fold change value below1.5), higher number of proteins were downregulated ( $n=1754)$ as compare to $12 \mathrm{hr}$ time point $(\mathrm{n}=247)$ post-infection condition (Table 1 , Fig. 2). This result indicates that most of the host proteins were downregulated at the later stage of infection.

Table 1

Differential protein expression analysis in chicken infected with HPAI H5N1 virus.

\begin{tabular}{|lllll|}
\hline Condition & $\begin{array}{l}\text { Upregulated } \\
\text { proteins }\end{array}$ & $\begin{array}{l}\text { Downregulated } \\
\text { proteins }\end{array}$ & $\begin{array}{l}\text { Upregulated } \\
\text { proteins } \\
(>1.5 \text { fold })\end{array}$ & $\begin{array}{l}\text { Downregulated } \\
\text { Proteins } \\
(<1.5 \text { fold })\end{array}$ \\
\hline $\begin{array}{l}12 \mathrm{hr} \\
\text { interval }\end{array}$ & 820 & 2493 & 138 & 247 \\
\hline $\begin{array}{l}24 \mathrm{hr} \\
\text { interval }\end{array}$ & 872 & 2441 & 157 & 222 \\
\hline $\begin{array}{l}48 \mathrm{hr} \\
\text { interval }\end{array}$ & 693 & 2620 & 173 & 1754 \\
\hline
\end{tabular}

Gene ontology analysis of the commonly upregulated and downregulated lung tissue proteins of H5N1 infected chicken lung enriched following GO terms such as cytoskeleton, regulation of cell cycle, regulation of protein kinase activity etc. (Table 2). The upregulation (KRT6A, MCPH1, MYH7, MICAL1 and MICAL3) and downregulation (ACTL9, CTNNB1, DNM1, FILIP1L, and MY01B) of cytoskeletal proteins were observed in the lung tissue of chicken. Cytoskeletal proteins have been reported to interact with viral proteins to regulate viral replication and assembly as well as the transport of viral components in the cell $[34,35]$. Similar associations of cytoskeletal proteins with influenza infection condition are also reported previously $[10,14,36]$. The cyclin-dependent kinases (CDKs) such as CDK13, DDB1, DRD3, FOXG1, and 
TCF3 that are involved in the regulation of cell cycle were all upregulated in chicken lung proteome. Söderholm et al. (2016) reported that the cyclin-dependent kinase activity is required for efficient viral replication and for activation of the host antiviral responses [37]. Protein kinase activity associated proteins, namely, JAK3, MARK3, TBK1, EIF2AK3, PRKCA, and TRPM6were downregulated in the chicken lung tissues. Differential expression of proteins including signal transduction molecules, kinases and other biochemical metabolism related enzymes that are associated with the repair of damaged lung tissues are reported in dogs infected with influenza virus infection [14]. In addition, some apoptosis and tumour-associated proteins (BCL6, FAF1, TBX5, AKAP13, TNFSF10 and TGFB1) were also identified in the chicken lung proteome. Further we did GO term analysis of proteins that were exclusively expressed in particular post-infection condition in chicken (Table 3). This analysis result provides information how the disease progress from onset of disease to outcome of disease in chicken. At $12 \mathrm{hr}$ interval, most of cellular homeostasis were affected and at $48 \mathrm{hr}$ post-infection condition the host activated critical pathways such as Influenza A, chemokine signaling pathway, Jak-STAT signaling pathway, apoptosis, MAPK signaling pathway (Table 3). These results indicate that the virus initially inhibit the cellular homeostasis and activates the critical pathways at the later stage of infection. 
Table 2

Gene Ontology term analysis of commonly upregulated and downregulated lung tissue proteins in chicken infected with HPAI H5N1 virus.

\begin{tabular}{|c|c|c|}
\hline GO terms & Count & P-Value \\
\hline \multicolumn{3}{|l|}{ Upregulated proteins } \\
\hline Golgi apparatus & 32 & 7.70E-04 \\
\hline Cellular homeostasis & 21 & 1.10E-03 \\
\hline Protein heterodimerization activity & 12 & $2.20 \mathrm{E}-03$ \\
\hline Ion homeostasis & 18 & 3.70E-03 \\
\hline Protein domain specific binding & 15 & $4.40 \mathrm{E}-03$ \\
\hline Acid-amino acid ligase activity & 11 & $5.20 \mathrm{E}-03$ \\
\hline Intracellular organelle lumen & 50 & $6.40 \mathrm{E}-03$ \\
\hline Fatty acid transport & 4 & $1.80 \mathrm{E}-02$ \\
\hline Regulation of cell cycle & 13 & $3.50 \mathrm{E}-02$ \\
\hline Vesicle-mediated transport & 19 & 4.20E-02 \\
\hline Positive regulation of protein kinase cascade & 8 & $5.10 \mathrm{E}-02$ \\
\hline Negative regulation of gene expression & 16 & 8.20E-02 \\
\hline Cytoskeleton & 35 & 8.60E-02 \\
\hline Adenyl nucleotide binding & 47 & $1.90 \mathrm{E}-03$ \\
\hline ATP binding & 43 & 4.90E-03 \\
\hline \multicolumn{3}{|l|}{ Downregulated proteins } \\
\hline Adenyl nucleotide binding & 236 & $1.20 \mathrm{E}-13$ \\
\hline ATP binding & 222 & $5.40 \mathrm{E}-13$ \\
\hline GTPase regulator activity & 74 & $4.50 \mathrm{E}-08$ \\
\hline Cytoskeleton & 176 & $1.20 \mathrm{E}-06$ \\
\hline Protein kinase activity & 82 & $9.20 \mathrm{E}-04$ \\
\hline Regulation of small GTPase mediated signal transduction & 47 & 8.10E-06 \\
\hline Regulation of Rho protein signal transduction & 25 & 1.10E-05 \\
\hline Proteinaceous extracellular matrix & 51 & 8.80E-05 \\
\hline Regulation of Ras protein signal transduction & 38 & $1.30 \mathrm{E}-04$ \\
\hline
\end{tabular}




\begin{tabular}{|lll|}
\hline GO terms & Count & P-Value \\
\hline Protein amino acid phosphorylation & 89 & $6.20 \mathrm{E}-04$ \\
\hline Cell death & 93 & $1.30 \mathrm{E}-03$ \\
\hline Enzyme linked receptor protein signalling pathway & 49 & $3.10 \mathrm{E}-03$ \\
\hline Endosome & 44 & $4.10 \mathrm{E}-03$ \\
\hline Intracellular signaling cascade & 146 & $4.30 \mathrm{E}-03$ \\
\hline Positive regulation of gene expression & 70 & $2.50 \mathrm{E}-02$ \\
\hline Calcium ion binding & 105 & $3.20 \mathrm{E}-02$ \\
\hline Negative regulation of translation & 8 & $3.60 \mathrm{E}-02$ \\
\hline Regulation of phosphorylation & 56 & $4.60 \mathrm{E}-02$ \\
\hline JUN kinase binding & 3 & $4.70 \mathrm{E}-02$ \\
\hline Proteolysis & 114 & $7.20 \mathrm{E}-02$ \\
\hline Positive regulation of MAP kinase activity & 15 & $9.60 \mathrm{E}-02$ \\
\hline
\end{tabular}


Table 3

Functional annotation analysis of proteins exclusively expressed in chicken at different time points post infection with HPAI H5N1 virus.

\begin{tabular}{|c|c|c|}
\hline Pathway activation & No. of proteins & P Value \\
\hline \multicolumn{3}{|l|}{$12 \mathrm{hr}$ post infection } \\
\hline Cell cycle & 31 & $2.88 \mathrm{E}-26$ \\
\hline mRNA surveillance pathway & 21 & 4.95E-18 \\
\hline RNA transport & 24 & $2.62 \mathrm{E}-17$ \\
\hline p53 signaling pathway & 11 & $1.22 \mathrm{E}-07$ \\
\hline Pathways in cancer & 13 & 0.0128 \\
\hline \multicolumn{3}{|l|}{$24 \mathrm{hr}$ post infection } \\
\hline Cell cycle & 12 & 1.17E-08 \\
\hline Gap junction & 8 & 7.31E-06 \\
\hline RNA degradation & 6 & $6.13 \mathrm{E}-05$ \\
\hline T cell receptor signaling pathway & 7 & 0.000127 \\
\hline Fc epsilon RI signaling pathway & 6 & 0.000215 \\
\hline ErbB signaling pathway & 6 & 0.000485 \\
\hline Phagosome & 4 & 0.00326 \\
\hline Fc gamma R-mediated phagocytosis & 5 & 0.00529 \\
\hline Chemokine signaling pathway & 7 & 0.00615 \\
\hline B cell receptor signaling pathway & 4 & 0.0112 \\
\hline Neurotrophin signaling pathway & 5 & 0.0141 \\
\hline Natural killer cell mediated cytotoxicity & 5 & 0.0221 \\
\hline Focal adhesion & 6 & 0.0287 \\
\hline \multicolumn{3}{|l|}{$48 \mathrm{hr}$ post infection } \\
\hline RNA transport & 47 & 1.77E-18 \\
\hline T cell receptor signaling pathway & 37 & $6.08 \mathrm{E}-15$ \\
\hline Regulation of actin cytoskeleton & 52 & $1.15 \mathrm{E}-14$ \\
\hline Focal adhesion & 54 & 4.67E-14 \\
\hline Pathways in cancer & 70 & $1.13 \mathrm{E}-13$ \\
\hline
\end{tabular}




\begin{tabular}{|lll|}
\hline Pathway activation & No. of proteins & P Value \\
\hline ErbB signaling pathway & 31 & $6.41 \mathrm{E}-12$ \\
\hline Adipocytokine signaling pathway & 26 & $6.92 \mathrm{E}-12$ \\
\hline B cell receptor signaling pathway & 28 & $1.91 \mathrm{E}-11$ \\
\hline Neurotrophin signaling pathway & 35 & $3.79 \mathrm{E}-10$ \\
\hline mRNA surveillance pathway & 26 & $6.14 \mathrm{E}-09$ \\
\hline Chemokine signaling pathway & 41 & $7.57 \mathrm{E}-08$ \\
\hline Influenza A & 28 & $1.68 \mathrm{E}-07$ \\
\hline Cell cycle & 30 & $3.95 \mathrm{E}-07$ \\
\hline Jak-STAT signaling pathway & 25 & $1.62 \mathrm{E}-06$ \\
\hline Fc epsilon RI signaling pathway & 21 & $1.86 \mathrm{E}-06$ \\
\hline mTOR signaling pathway & 14 & $2.72 \mathrm{E}-05$ \\
\hline Toll-like receptor signaling pathway & 20 & 0.000377 \\
\hline Apoptosis & 18 & 0.000385 \\
\hline Natural killer cell mediated cytotoxicity & 24 & 0.00139 \\
\hline MAPK signaling pathway & 38 & 0.00297 \\
\hline Leukocyte transendothelial migration & 19 & 0.00374 \\
\hline RIG-I-like receptor signaling pathway & 11 & 0.0039 \\
\hline
\end{tabular}

\section{Molecular pathogenesis of H5N1 infection in chicken}

Previous transcriptomics study of our group found that highly pathogenic H5N1 virus induced excessive expression of type I IFNs, cytokine, chemokines and ISGs in the lung tissues. This atypical expression of immune genes (cytokine storm) might be the cause for the high mortality in chickens [28]. However, the information on the pathways activated, constituents of cytokine storm and therapeutic strategies against cytokine storm is lacking in avian species. Intensive molecular studies in humans and human animal models system have identified, (1) Activation of TLR3 and 7, as well as endosome (TLR3 and 7) and cytosolic (RIG-I) pathways; (2) activation of IL-1R signaling pathway; and (3)activation of MVAS/MyD88/TRIF signaling as essential pathways involved in the cytokine storm [38].

In order to check whether these pathways are activated and if activated what are the levels of expression of the immune genes in chicken lung tissues, we applied meta-analysis of lung transcriptome datasets. We utilised the previously published microarray datasets of chicken infected with HPAIVs, because transcriptomic data can capture the complete gene expression dynamics at a particular condition. We 
mapped the differentially expressed genes retrieved from meta-analysis results of chicken into influenza reference pathways in KEGG database. Activation of TLR signaling pathway, RIG I signaling pathway, NOD like receptor signaling pathway and JAK-STAT signaling pathway were observed in chicken lung transcriptome (Fig.S2). Similarly, activation of these pathways was evident in the chicken proteome datasets (Fig. 3). Further Bayesian networks (BN) were constructed with prior knowledge using chicken meta-analysis transcriptome datasets (Fig. 4, Fig. S3). The combined in silico analysis of transcriptome and proteome datasets confirmed the activation of TLR, RLR, NLR and Jak-STAT signaling pathways in lung tissues infected with HPAIVs in chickens. The fact that most of the influenza pathogenesis possesses abnormalities in all of these core pathways suggests they play a central role in the cytokine storm.

Next, we examined the cytokine storm responsive genes (i.e expression level of cytokines, chemokines and ISGs) as a result of activation of the above-mentioned pathways. Cytokine storm responsive genes lists were compiled for chickens based on information in literature [2]. The expression levels (fold change) of these genes were retrieved from meta-analysis transcriptome datasets of chicken. Cytokines, chemokines and ISGs genes were found to be upregulated in chicken lung tissues and these may be the basis of the increase in the severity of HPAI H5N1 infection in chickens (Fig. 5). In summary, we characterized the immune pathways involved in the cytokine storm and identified cytokine storm responsive genes in chicken lung tissues infected with HPAIVs.

\section{Identification of proteomic determinants responsible for disease pathogenesis in chicken}

To know the main driver or hub proteins responsible for disease pathogenesis, we constructed proteinprotein interaction (PPI) network based on chicken lung proteome dataset (Fig. 6). The proteins such as MyD88, IKBKB, IRAK4, RELA, and MAVS involved in the TLRs, RLRs, IL1R and NLR signaling pathways were identified with high degree centrality and betweenness centrality measures (Table 4). 
Table 4

Hub proteins identified in chicken PPI networks based on degree centrality and betweenness centrality measures.

\begin{tabular}{|lll|}
\hline Proteins & Degree centrality & Betweenness centrality \\
\hline HNF4A & 254 & 254342.21 \\
\hline ELAVL1 & 254 & 253172.78 \\
\hline FN1 & 117 & 79642.94 \\
\hline COPS5 & 117 & 73208.97 \\
\hline CUL1 & 105 & 55049.71 \\
\hline CAND1 & 105 & 45936.7 \\
\hline CTNNB1 & 80 & 75126.54 \\
\hline BRCA1 & 72 & 41422.24 \\
\hline FYN & 65 & 35789.72 \\
\hline MYD88 & 10 & 2614.04 \\
\hline IKBKB & 42 & 16555.52 \\
\hline RELA & 48 & 23497.55 \\
\hline MAVS & 6 & 1721.53 \\
\hline STAT1 & 81 & 46280.33 \\
\hline STAT2 & 14 & 1579.16 \\
\hline STAT3 & 90 & 66853.77 \\
\hline SOCS3 & 23 & 11524.2 \\
\hline IRAK4 & 8 & 311.94 \\
\hline
\end{tabular}

MYD88 gene encodes a cytosolic adapter protein that plays a central role in the innate and adaptive immune response. This protein functions as an essential signal transducer in the IL1R and TLR signaling pathways. These pathways in turn regulate the activation of numerous proinflammatory genes [39]. The IKBKB protein phosphorylates the inhibitor in the inhibitor/NF-kappa-B complex, causing dissociation of the inhibitor and activation of NF-kB signaling pathway [40]. MAVS acts downstream of DDX58/RIG-I and IFIH1/MDA5 genes, as an essential signal transducer in the beta interferon signaling pathways and that contribute to antiviral immunity [41]. RELA/NF-kB is a ubiquitous transcription factor involved in several biological processes. This transcription factor is activated through degradation of its specific inhibitor in the cytoplasm; NF-kB moves to the nucleus and activates transcription of specific genes. The NF-kBp65p65 complex appears to be involved in invasin-mediated activation of IL-8 expression [42]. Teijaro et al. (2014) reported that MyD88 and MAVS as the predominant signaling molecule required for innate 
immune cell recruitment and for the majority of cytokine amplification (i.e., cytokine storm) in mice infected with influenza virus [38]. Further they suggested that therapeutic control of cytokine storm is possible through a common pathway inhibition downstream of multiple innate pathogen-sensing molecules of cytokine amplification. In our study the identified hub proteins (MyD88, IKBKB, IRAK4, RELA, and MAVS) were all involved in different components of MyD88 and MVAS signaling pathways. Based on this literature, we suggest that successful therapeutic intervention for cytokine storm in chicken should target these proteins as drug targets to blunt the cytokine amplification. Further the S1P1R agonist therapy may suppress global cytokine amplification in chicken as in mice [38]. However biological validation of this hypothesis by in vivo experiment is needed.

In Jak-STAT signalling pathway, we found STAT1, STAT2, STAT3 and SOCS3 proteins as the main driving proteins in the PPI network (Table 4). However, these proteins had protein probability range from 0.63 to 0.90 , hence were not evident in our main proteome dataset of chicken. The STAT1, STAT2 and STAT-3 proteins are a key constituents of JAK-STAT signalling pathway, play critical roles in the IFN signalling pathway and are required for a robust IFN-induced antiviral response [43, 44]. SOCS1 and SOCS3 are reported to be critical regulators of IFN responses through the inhibition of STAT phosphorylation and induction of ISGs through a RIG-I/MAVS/IFNAR1-dependent pathway [45, 46].

Further we identified some novel main driver/hub proteins with a very high degree of centrality and betweenness of centrality measures which are not reported previously to be associated with influenza infection condition in human, human models or avian species. These novel hub proteins are HNF4A, ELAVL1, FN1, COPS5, CUL1, CAND1, BRCA1, CTNNB1 and FYN (Table 4, Fig. 6). The HNF4A protein is a transcriptionally controlled transcription factor and required for the transcription of alpha 1-antitrypsin, apolipoprotein CIII, transthyretin genes and HNF1-alpha genes [47]. ELAVL1 is a member of the ELAVL family of RNA-binding proteins and selectively bind AU-rich elements (AREs) found in the $3^{\prime}$ untranslated regions of mRNAs. The ELAVL family of proteins play a role in stabilizing ARE-containing mRNAs [48]. This gene has been implicated in a variety of biological processes and has been linked to a number of diseases, including cancer [49].

FN1 bind cell surfaces and various compounds including collagen, fibrin, heparin, DNA, and actin. FN1 is involved in cell adhesion and migration processes during embryogenesis, wound healing, blood coagulation, host defense, and metastasis [50]. COPS5 is one of the eight subunits of COP9 signalosome and functions as an important regulator in phosphorylation of p53/TP53, c-jun/JUN, IkappaBalpha/NFKBIA, ITPK1 and IRF8 signalling [51]. CUL1 is a core component of multiple cullin-RINGbased SCF (SKP1-CUL1-F-box protein) E3 ubiquitin-protein ligase complexes, which mediate the ubiquitination of proteins involved in cell cycle progression, signal transduction and transcription [52].

BRCA1 encodes a nuclear phosphoprotein that has a role in maintaining genomic stability, and acts as a tumour suppressor. This protein is involved in transcription, DNA repair of double-stranded breaks, and recombination [53]. FYN gene encodes a membrane-associated tyrosine kinase that has been implicated in the control of cell growth [54]. In summary, many proteins involved in the TLRs, RLRs, NLR and Jak- 
STAT signaling pathways and other novel proteins were identified as main protein determinant and these proteins might be linked with disease pathogenesis in chicken H5N1 infection. However critical functional role of these proteins in avian influenza pathogenesis in chicken requires further biological validation by in vivo and in vitro experiments.

\section{Conclusion}

In conclusion, we characterized the comprehensive proteome profile of chicken lung tissues infected with HPAI H5N1 virus at different time points post-infection. There are differences in the protein profile at different time points post-infection indicated by the remarkable variations in the levels of expression of proteins at these time points. Combined analysis of transcriptome and proteome datasets revealed activation of TLR, RLR, NLR, and JAK-STAT signaling pathways associated with a cytokine storm effect in chicken infected with HPAI H5N1 virus. Further, we identified many of the important hub proteins linked with influenza pathogenesis in chicken.

\section{Declarations}

\section{Ethics statement}

The experiments were approved by the Institutional Animal Ethics Committee of ICAR-NIHSAD (Approval no. 68/IAEC/HSADL/12 dated 11.05.2012), and performed under the guidance of the Committee for the Purpose of Control and Supervision of Experiments on Animals (CPCSEA), Ministry of Environment and Forests, Govt. of India.

\section{Availability of data and materials}

The mass spectrometry proteomics data have been deposited to the ProteomeXchange Consortium via the PRIDE partner repository with the dataset identifier PXD010358.

\section{Competing Interests}

The authors declare no competing interests.

\section{Funding}

This work was funded by the Department of Biotechnology (grant number: BT/IN/IndoUK/FADH/48/AM/2013). The funders had no role in study design, data collection, analysis of data and preparation of the manuscript.

\section{Author Contributions}

Conceived and designed the experiments: A.M., A.A.R. and P.V. Performed the experiments: A.M., S.C., A.A.R and P.V. Analyzed the data: P.V. and A.M. Contributed reagents/materials/analysis tools: H.V.M., 
D.D.K. and V.P.S Wrote the paper: P.V. and A.M. All authors have read and approved the manuscript.

\section{Acknowledgements}

We thank Director, ICAR-National Institute of High Security Animal Diseases, Director, ICAR-Indian Veterinary Research Institute, and Indian Council of Agricultural Research, India for providing necessary facilities to carry out this work.

\section{References}

1. Fauci AS (2006) Emerging and re-emerging infectious diseases: influenza as a prototype of the hostpathogen balancing act. Cell 24:665-670

2. Mishra A, Vijayakumar P, Raut AA (2017) Emerging avian influenza infections: Current understanding of innate immune response and molecular pathogenesis. Int Rev Immunol 236:89-107

3. Josset L, Tisoncik Go J, Katze MG (2013) Moving H5N1 studies into the era of systems biology. Virus Res 178:151-167

4. Zak DE, Tam VC, Aderem A (2014) Systems-level analysis of innate immunity. Annu Rev Immunol 32:547-577

5. Gingras AC, Gstaiger M, Raught B, Aebersold R (2007) Analysis of protein complexes using mass spectrometry. Nature Rev Mol Cell Biol 8:645-654

6. Altelaar AF, Munoz J, Heck AJ (2013) Next-generation proteomics: towards an integrative view of proteome dynamics. Nat Rev Genet 14:35-48

7. Brown JN et al (2010) Macaque proteome response to highly pathogenic avian influenza and 1918 reassortant influenza virus infections. J Virol 84:12058-12068

8. Kumar $Y$ et al (2014) Molecular analysis of serum and bronchoalveolar lavage in a mouse model of influenza reveals markers of disease severity that can be clinically useful in humans. PLoS One 9:e86912

9. Vester D, Rapp E, Gade D, Genzel Y, Reichl U (2009) Quantitative analysis of cellular proteome alterations in human influenza A virus-infected mammalian cell lines. Proteomics 9:3316-3327

10. Coombs KM et al (2010) Quantitative proteomic analyses of influenza virus-infected cultured human lung cells. J Virol 84:10888-10906

11. Kummer S et al (2014) Alteration of protein levels during influenza virus H1N1 infection in host cells: a proteomic survey of host and virus reveals differential dynamics. PLoS One 9:e94257

12. Zou W et al (2014) Proteomics analysis of differential expression of chicken brain tissue proteins in response to the neurovirulent H5N1 avian influenza virus infection. J Proteome Res 9:3789-3798

13. Li Y et al (2017) Proteome Response of Chicken Embryo Fibroblast Cells to Recombinant H5N1 Avian Influenza Viruses with Different Neuraminidase Stalk. Lengths Sci Rep 7:40698

14. Su S et al (2015) Global and quantitative proteomic analysis of dogs infected by avian-like H3N2 canine influenza virus. Front Microbiol 6:228 
15. Liu N et al (2008) Proteomics analysis of differential expression of cellular proteins in response to avian H9N2 virus infection in human cells. Proteomics 8:1851-1858

16. Lietzen $\mathrm{N}$ et al (2011) Quantitative subcellular proteome and secretome profiling of influenza A virusinfected human primary macrophages. PLoS Pathog 7:e1001340

17. Kroeker AL, Ezzati P, Halayko AJ, Coombs KM (2012) Response of primary human airway epithelial cells to influenza infection: a quantitative proteomic study. J Proteome Res 11:4132-4146

18. Liu L, Zhou J, Wang Y, Mason RJ, Funk CJ, Du Y (2012) Proteome alterations in primary human alveolar macrophages in response to influenza A virus infection. J Proteome Res 11:4091-4101

19. Chambers MC et al (2012) A cross-platform toolkit for mass spectrometry and proteomics. Nat Biotechnol 30:918-920

20. Deutsch EW et al (2010) A guided tour of the Trans-Proteomic Pipeline. Proteomics 10:1150-1159

21. Eng JK, Jahan TA, Hoopmann MR (2013) Comet: an open-source MS/MS sequence database search tool. Proteomics 13:22-24

22. Keller A, Nesvizhskii Al, Kolker E, Aebersold R (2002) Empirical statistical model to estimate the accuracy of peptide identifications made by MS/MS and database search. Anal Chem 74:53835392

23. Nesvizhskii Al, Aebersold R (2004) Analysis, statistical validation and dissemination of large-scale proteomics datasets generated by tandem MS. Drug Discov Today 9:173-181

24. Huang DW, Sherman BT, Lempicki RA (2009) Systematic and integrative analysis of large gene lists using DAVID Bioinformatics Resources. Nature Protoc 4:44-57

25. Metsalu T, Vilo J (2015) ClustVis: a web tool for visualizing clustering of multivariate data using Principal Component Analysis and heatmap. Nucleic Acids Res 43:W566-W570

26. Xia J, Benner MJ, Hancock RE (2014) NetworkAnalyst-integrative approaches for protein-protein interaction network analysis and visual exploration. Nucleic Acids Res 42:W167-W174

27. Hu J et al (2015) PA-X decreases the pathogenicity of highly pathogenic H5N1 influenza A virus in avian species by inhibiting virus replication and host response. J Virol 89:4126-4142

28. Ranaware B et al (2016) Genome Wide Host Gene Expression Analysis in Chicken Lungs Infected with Avian Influenza Viruses. PLoS One 11:e0153671

29. Scutari M (2017) Bayesian Network Constraint-Based Structure Learning Algorithms: Parallel and Optimized Implementations in the bnlearn R Package. J Stat Softw 77:1-20

30. Wagner R, Matrosovich M, Klenk HD (2002) Functional balance between haemagglutinin and neuraminidase in influenza virus infections. Rev Med Virol 12:159-166

31. Portela A, Digard P (2002) The influenza virus nucleoprotein: a multifunctional RNA-binding protein pivotal to virus replication. J Gen Virol 83:723-734

32. Wasilenko JL et al (2008) NP, PB1, and PB2 viral genes contribute to altered replication of H5N1 avian influenza viruses in chickens. J Virol 82:4544-4553 
33. Hulse-Post DJ et al (2007) Molecular changes in the polymerase genes PA and PB1 associated with high pathogenicity of H5N1 influenza virus in mallard ducks. J Virol 81:8515-8524

34. Avalos RT, Yu Z, Nayak DP (1997) Association of influenza virus NP and M1 proteins with cellular cytoskeletal elements in influenza virus-infected cells. J Virol 71:2947-2958

35. Radtke K, Dohner K, Sodeik B (2006) Viral interactions with the cytoskeleton: a hitchhiker's guide to the cell. Cell Microbiol 8:387-400

36. Sui Z, Wen B, Gao Z, Chen Q (2014) Fusion-Related Host Proteins Are Actively Regulated by NA during Influenza Infection as Revealed by Quantitative Proteomics Analysis. PLoS ONE 9:e105947

37. Soderholm S et al (2016) Phosphoproteomics to characterize host response during influenza A virus infection of human macrophages. Mol Cell Proteomics 15:3203-3219

38. Teijaro JR, Walsh KB, Rice S, Rosen H, Oldstone MB (2014) Mapping the innate signaling cascade essential for cytokine storm during influenza virus infection. Proc Natl Acad Sci U S A 111:37993804

39. Deguine J, Barton GM (2014) MyD88: a central player in innate immune signaling. F1000Prime Rep 4:6:97

40. Rothwarf DM, Karin M (1999) The NF-kappa B activation pathway: a paradigm in information transfer from membrane to nucleus. Sci STKE, RE1

41. Seth RB, Sun L, Ea CK, Chen ZJ (2005) Identification and characterization of MAVS, a mitochondrial antiviral signaling protein that activates NF-kappaB and IRF 3. Cell 122:669-682

42. Kunsch C, Rosen CA (1993) NF-kappa B subunit-specific regulation of the interleukin-8 promoter. Mol Cell Biol 13:6137-6146

43. Yang CH, Murti A, Pfeffer LM (1998) STAT3 complements defects in an interferon-resistant cell line: evidence for an essential role for STAT3 in interferon signaling and biological activities. Proc Natl Acad Sci U S A 95:5568-5572

44. Ho HH, Ivashkiv LB (2006) Role of STAT3 in type I interferon responses Negative regulation of STAT1-dependent inflammatory gene activation. J Biol Chem 281:14111-14118

45. Pauli EK et al (2008) Influenza A virus inhibits type I IFN signaling via NF-KB dependent induction of SOCS-3 expression. PLoS Pathog 4:e1000196

46. Pothlichet J, Chignard M, Si-Tahar M (2008) Cutting edge: innate immune response triggered by influenza A virus is negatively regulated by SOCS1 and SOCS3 through a RIG-I/IFNAR1-dependent pathway. J Immunol 180:2034-2038

47. Ryffel GU (2001) Mutations in the human genes encoding the transcription factors of the hepatocyte nuclear factor HNF 1 and HNF4 families: functional and pathological consequences. $J$ Mol Endocrinol 27:11-29

48. Brennan CM, Steitz JA (2001) HuR and mRNA stability. Cell Mol Life Sci 58:266-277

49. Wang $\mathrm{H}$ et al (2013) The structure of the ARE-binding domains of Hu antigen $\mathrm{R}$ HuR undergoes conformational changes during RNA binding. Acta Crystallogr D Biol Crystallogr 69:373-380 
50. Liao YX, Zhang ZP, Zhao J, Liu JP (2018) Effects of Fibronectin 1 on Cell Proliferation, Senescence and Apoptosis of Human Glioma Cells Through the PI3K/AKT Signaling Pathway. Cell Physiol Biochem 48:1382-1396

51. Bech-Otschir D, Seeger M, Dubiel W (2002) The COP9 signalosome: at the interface between signal transduction and ubiquitin-dependent proteolysis. J Cell Sci 115:467-473

52. Kipreos ET et al (1996) cul-1 is required for cell cycle exit in C elegans and identifies a novel gene family. Cell 85:829-839

53. Foulkes WD, Shuen AY (2013) In brief: BRCA1 and BRCA2. J Pathol 230:347-349

54. Zheng J, Li H, Xu D, Zhu H (2017) Upregulation of Tyrosine Kinase FYN in Human Thyroid Carcinoma: Role in Modulating Tumor Cell Proliferation, Invasion, and Migration. Cancer BiotherRadiopharm 32:320-326

\section{Figures}

a)

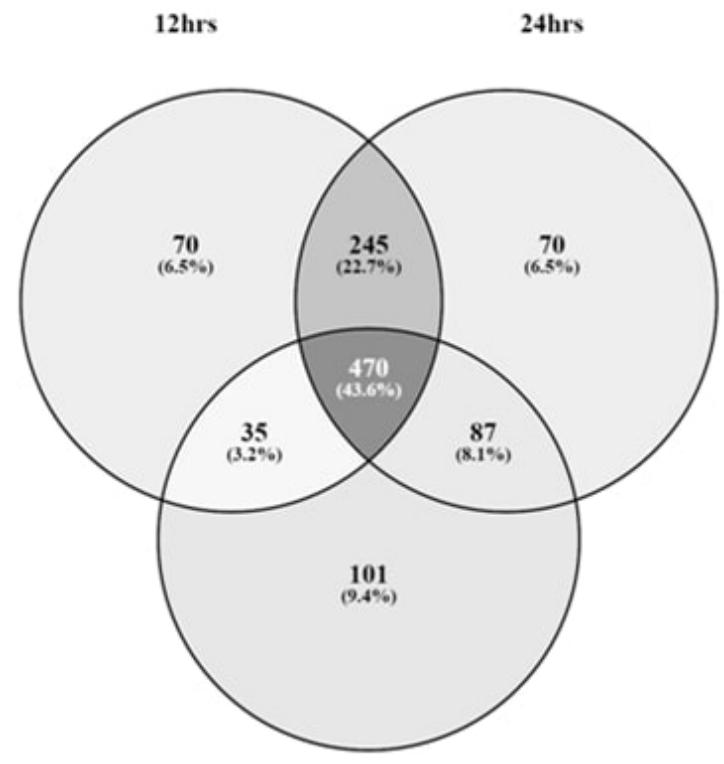

48hrs b)

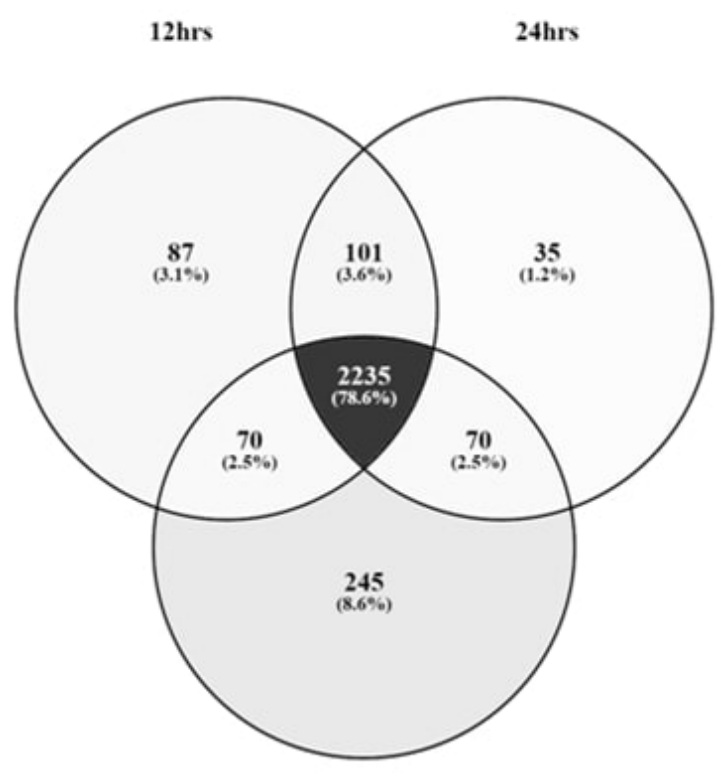

48hrs

\section{Figure 1}

The protein profile showed that 70,70 and 101 proteins were exclusively up regulated in chicken lung tissue at $12 \mathrm{hr}, 24 \mathrm{hr}$ and $48 \mathrm{hr}$ post-infection, respectively. Downregulation of 87,35 and 245 proteins were observed exclusively at $12 \mathrm{hr}, 24 \mathrm{hr}$ and $48 \mathrm{hr}$ post-infection, respectively 


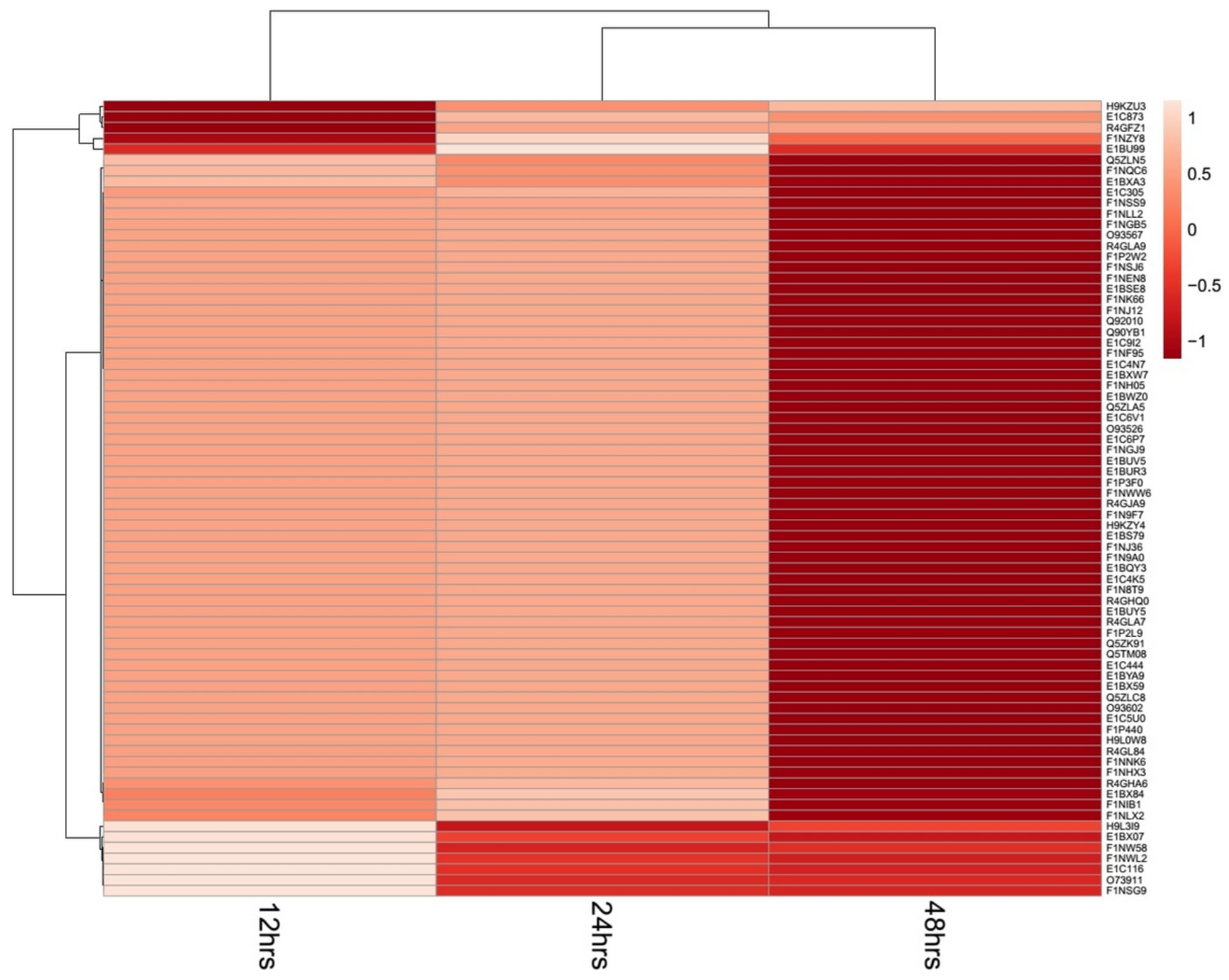

Figure 2

The fold change value of the upregulated proteins ranged from 42 to 1 . Interestingly, at $48 \mathrm{hr}$ time point (fold change value below1.5), higher number of proteins were downregulated $(n=1754)$ as compare to 12 hr time point ( $\mathrm{n}=247)$ post-infection condition (Table 1) 


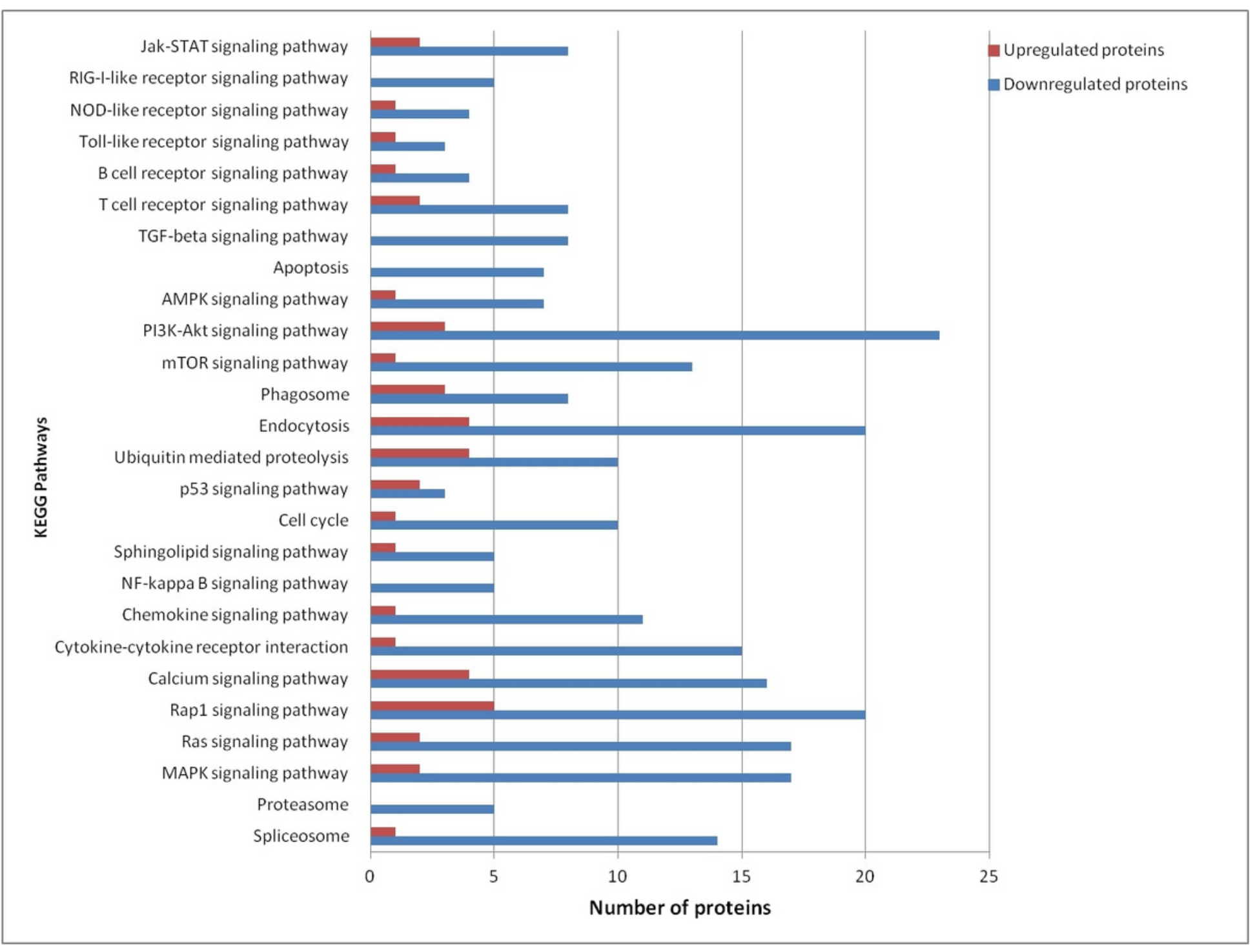

Figure 3

Activation of these pathways was evident in the chicken proteome datasets. 
TRIM25

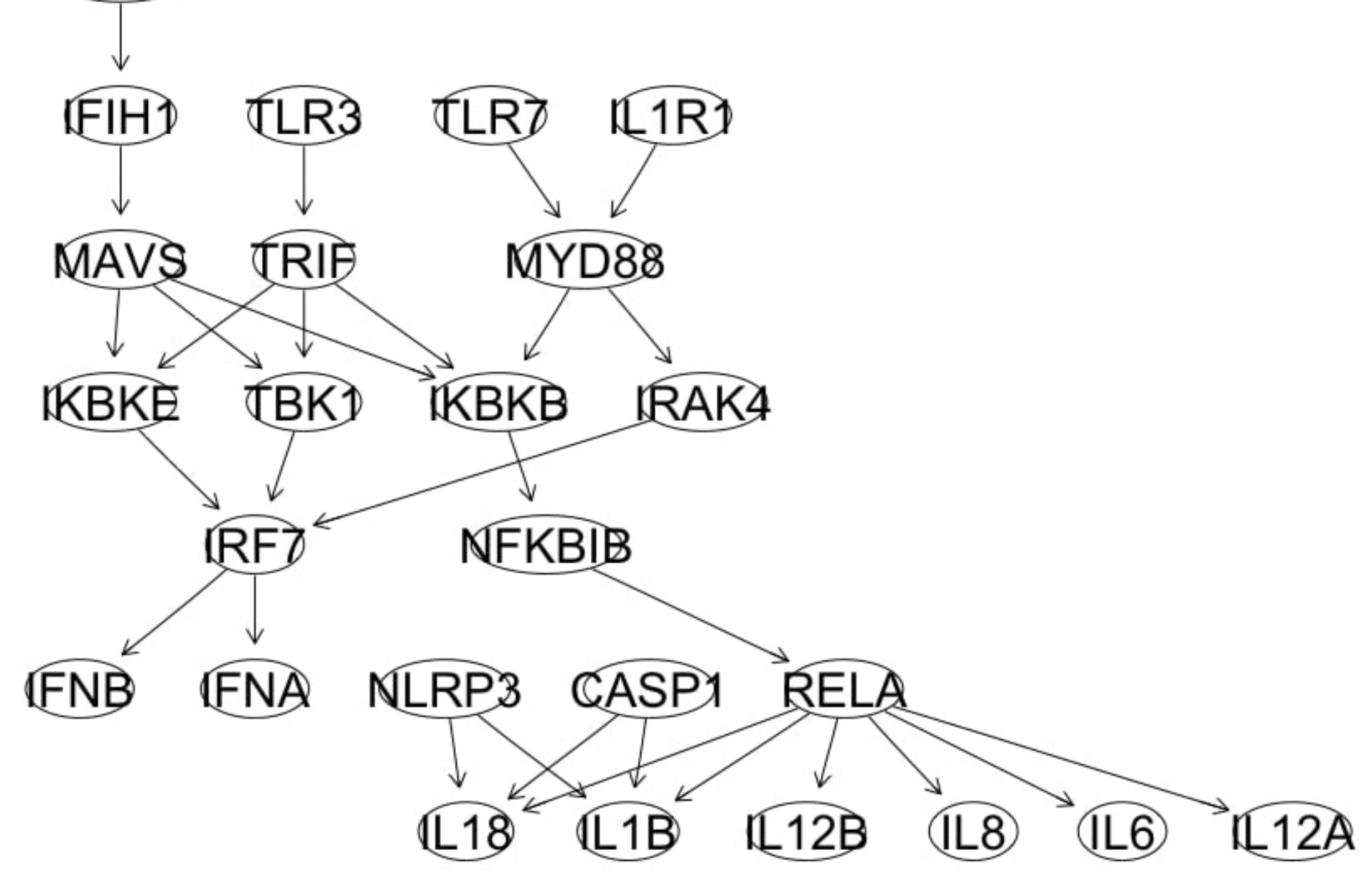

Figure 4

Bayesian networks (BN) were constructed with prior knowledge using chicken meta-analysis transcriptome datasets. 


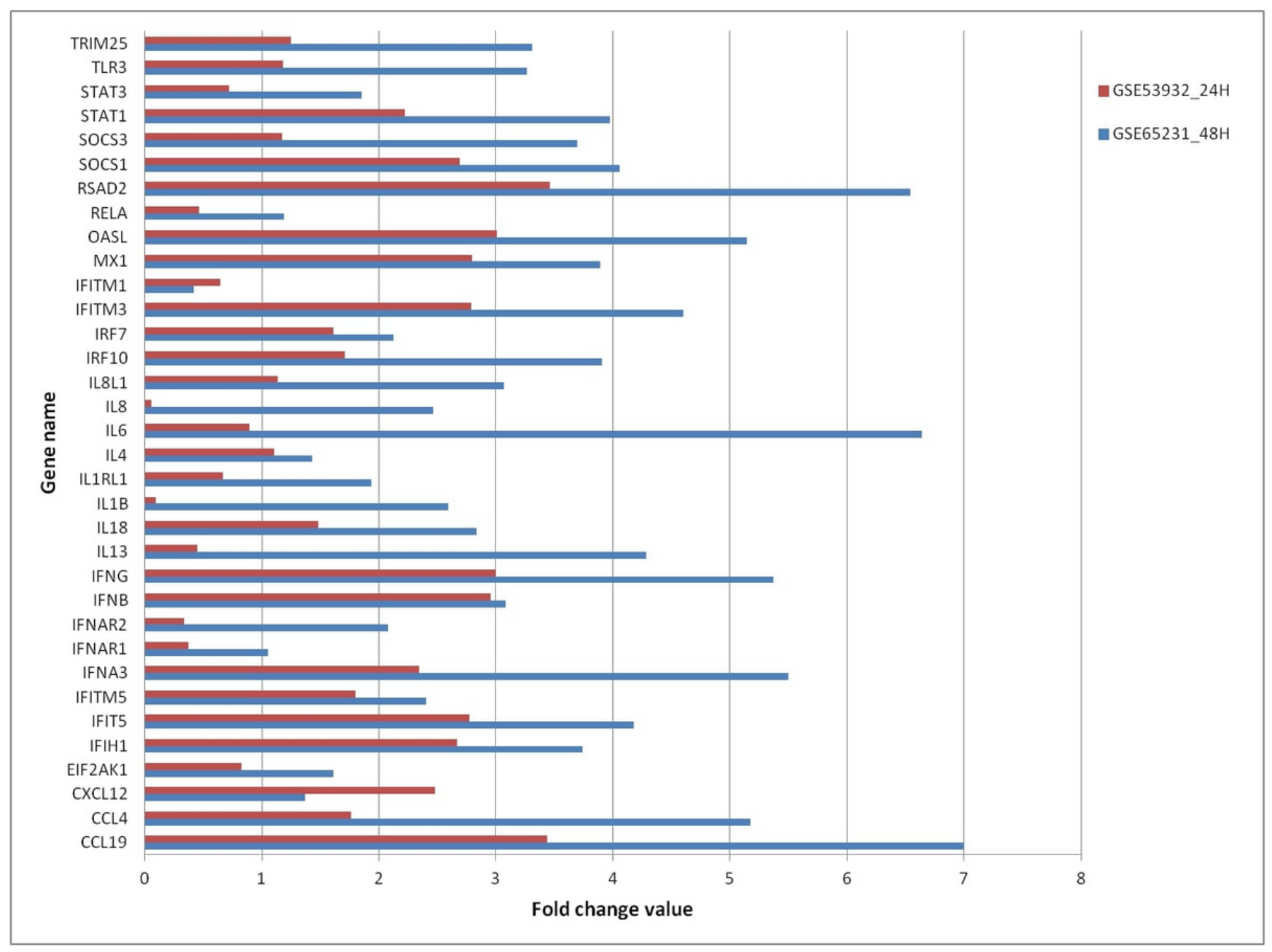

Figure 5

The expression levels (fold change) of these genes were retrieved from meta-analysis transcriptome datasets of chicken. Cytokines, chemokines and ISGs genes were found to be upregulated in chicken lung tissues and these may be the basis of the increase in the severity of HPAI H5N1 infection in chickens . 


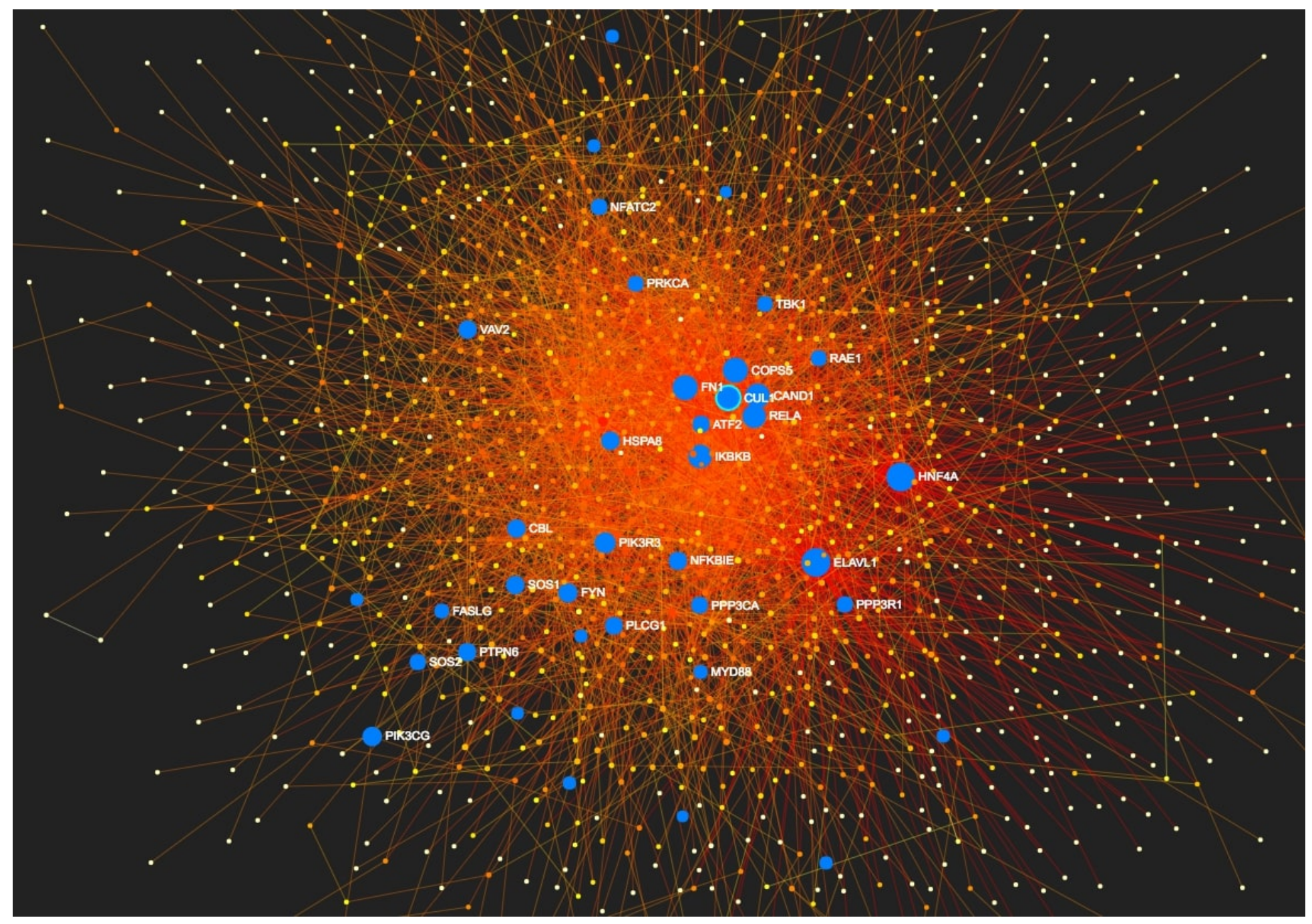

Figure 6

To know the main driver or hub proteins responsible for disease pathogenesis, we constructed proteinprotein interaction (PPI) network based on chicken lung proteome dataset .

\section{Supplementary Files}

This is a list of supplementary files associated with this preprint. Click to download.

- Supplementaryinformation.docx 